SLAC-TN-10-089

LCLS-TN-05-09

\title{
Temperature Measurements In The Magnetic Measurement Facility
}

\author{
Zachary Wolf, Vsevolod Kaplounenko \\ SLAC
}

April 5, 2005

\begin{abstract}
All the LCLS undulators must be tuned in the Magnetic Measurement Facility at the same temperature to within $\pm 0.1^{\circ} \mathrm{C}$. In order to ensure this, we are building a system to measure the temperature of the undulators to $\pm 0.01^{\circ} \mathrm{C}$. This note describes the temperature measurement system under construction.
\end{abstract}

\section{Introduction}

Several key LCLS undulator parameter values depend strongly on temperature primarily because of the permanent magnet material the undulators are constructed with. The undulators will be tuned to have specific parameter values in the Magnetic Measurement Facility (MMF). Consequently, it is necessary for the temperature of the MMF to remain fairly constant. Requirements on undulator temperature have been established. When in use, the undulator temperature will be in the range $20.0 \pm 0.2^{\circ} \mathrm{C}^{1}$. In the MMF, the undulator tuning will be done at $20.0 \pm 0.1^{\circ} \mathrm{C}^{2}$. For special studies, the MMF temperature set point can be changed to a value between $18^{\circ} \mathrm{C}$ and $23^{\circ} \mathrm{C}$ with stability of $\pm 0.1^{\circ} \mathrm{C}$. In order to ensure that the MMF temperature requirements are met, the MMF must have a system to measure temperatures. The accuracy of the MMF temperature measurement system must be better than the $\pm 0.1^{\circ} \mathrm{C}$ undulator tuning temperature tolerance, and is taken to be $\pm 0.01^{\circ} \mathrm{C}$.

The temperature measurement system for the MMF is under construction. It is similar to a prototype system we built two years ago in the Sector 10 alignment lab at SLAC. At that time, our goal was to measure the lab temperature to $\pm 0.1^{\circ} \mathrm{C}$. The system has worked well for two years and has maintained its accuracy. For the MMF system, we propose better sensors and a more extensive calibration program to achieve the factor of 10 increase in accuracy.

In this note we describe the measurement system under construction. We motivate our choice of system components and give an overview of the system. Most of the software for

\footnotetext{
${ }^{1}$ Heinz-Dieter Nuhn et al., "General Undulator System Requirements", LCLS Physics Requirements Document 1.4-001.

${ }^{2}$ R. Ruland et al., "Magnetic Measurements Facility Requirements", LCLS Physics Requirements Document 1.4-002.

This work was performed in support of the LCLS project at SLAC
}

Work supported in part by US Department of Energy contract DE-AC02-76SF00515. 
the system has been written and will be discussed. We discuss error sources in temperature measurements and show how these errors have been dealt with. The calibration system is described in detail.

\section{Overview of the Temperature Measurement System}

\subsection{Motivation}

The tight temperature tolerance of the MMF is easy to motivate. The remnant field of permanent magnet material typically changes by about $0.1 \%$ per degree Celsius ${ }^{3}$. The $K$ value of each undulator, which depends on the magnetic field strength, must be set to $0.015 \% \%^{4}$. Thus, a temperature change of $0.15^{\circ} \mathrm{C}$ will use up the entire tolerance on $K$. In practice, the undulators have some compensation built in ${ }^{5}$ which allows the temperature tolerance to be as large as it is.

A high quality air conditioning system will keep the MMF air temperature within tolerance. When an undulator is brought into the MMF, it will take several days to come to thermal equilibrium at the air temperature. During this time, the temperature will be monitored by the system under construction. When the undulators are placed on the test bench for magnetic measurements, the temperature must be accurately recorded. This will also be done with the system under construction. Finally, the system will monitor the MMF ambient temperature to insure that the air conditioning system is keeping the air temperature within its tolerance.

\subsection{Required System Features}

The primary requirement for the MMF temperature measurement system is the measurement accuracy of $\pm 0.01^{\circ} \mathrm{C}$. It should be noted that even though the FEL physics only requires that the undulators be the same and thus be tuned at the same temperature, the temperature measurement requirement must be on accuracy and not on precision. The measurements will take place over many years with possible instrument failure, reference probe failure, etc. The only way to guarantee high precision is to have high accuracy.

The temperature measurement system must be capable of monitoring a number of temperatures. The MMF will have a region encompassing the test stands in which roughly 10 ambient temperature measurements are desired. There will typically be four undulators in the MMF, two under test and two coming to thermal equilibrium. Each undulator will have four temperature sensors on it, making a total of 16 temperature measurements for the undulators. In total, we require 26 temperatures to be continuously monitored. In addition, the system should have an atmospheric pressure measurement, relative humidity measurement, and a reference resistor measurement. We thus seek a system capable of continuously monitoring nearly 30 signals.

\footnotetext{
${ }^{3}$ Data from http://www.magnetsales.com/Neo/Neoprops.htm, Magnet Sales \& Manufacturing Inc., Culver City, California.

${ }^{4}$ Heinz-Dieter Nuhn et al., "General Undulator System Requirements", LCLS Physics Requirements Document 1.4-001.

${ }^{5}$ R. Dejus (Editor), "LCLS Prototype Undulator Report", ANL/APS/TB-48, January, 2004.
} 
The overall system must include means to calibrate sensors and check for errors. Because there are a large number of sensors requiring routine calibration, we decided to calibrate inhouse rather than send the sensors to a calibration laboratory. Our calibration techniques and error checks will be described in detail below.

\subsection{Choice of System Components}

There are many choices one must make when developing a temperature measurement system. The first choice is between a totally commercial system and a system assembled out of commercial instruments and sensors. We chose the assembled system based on cost. Commercial instruments with an accuracy of $\pm 0.01^{\circ} \mathrm{C}$ are expensive and since we require 26 temperature measurements, the cost is high. It is not clear that a lot is gained from this approach either: software must still be written to collect the temperature measurements, the probes must still be calibrated periodically. Instead, we chose to build the system using a DMM, a multiplexer, temperature sensors, and calibration equipment. The system is low cost, flexible, tailored to our needs, and provides easy calibration checks.

The biggest choice in the system assembled from instruments and sensors is sensor type. There are many types of sensors: RTD, thermistor, thermocouple, semiconductor, and others which are somewhat application specific such as optical and acoustic sensors ${ }^{6}$. Each sensor type has advantages and disadvantages including the range of operating temperature, sensitivity, long term stability, electrical interface, and allowed environmental conditions. The MMF will have very benign environmental conditions, a very small temperature range, and requires high accuracy. We seek a sensor with excellent long term stability and high sensitivity. Based on this criterion, thermistors are the obvious choice. High grade thermistors have sensitivities of over $4 \%$ resistance change per degree Celsius and long term stability better than $0.01^{\circ} \mathrm{C}$ over 100 months $^{7}$. Of the other choices, thermocouples have very low sensitivity, for example the sensitivity of Copper/Constantan is $4 \times 10^{-5} \mathrm{~V} /{ }^{\circ} \mathrm{C}^{8}$, making signal processing for accurate temperature measurements very difficult. Semiconductor sensors do not have the required accuracy. They have self heating errors larger than our requirements ${ }^{9}$. RTDs are the temperature sensor of choice when accuracy over a large temperature range is required. They are fragile and have low sensitivity, however, requiring much care in their use ${ }^{10}$. The low sensitivity of an RTD is a big disadvantage when the range of temperatures is very small and the accuracy requirement is very high. In contrast, thermistors have very high sensitivity, excellent long term stability, and can be calibrated for highly accurate measurements. They are the best choice for our application.

Many companies make thermistors, necessitating the next choice. Thermometrics, Betatherm, YSI, and others make excellent products. We require the thermistor to be mounted in a probe package making our order semi-custom. Of the three companies

\footnotetext{
${ }^{6}$ J. Fraden, AIP Handbook of Modern Sensors, American Institute of Physics, 1993.

${ }^{7}$ See, for instance, the specifications for YSI 46000 series thermistors at www.ysi.com.

${ }^{8}$ J. Fraden, AIP Handbook of Modern Sensors, American Institute of Physics, 1993.

${ }^{9}$ See, for instance, "LM35 Precision Centigrade Temperature Sensor" from National Instruments, Santa Clara, CA. The self heating error is $0.08^{\circ} \mathrm{C}$ in air. The device has a specified accuracy of $1^{\circ} \mathrm{C}$, but some of this error could be calibrated out.

${ }^{10}$ A. Svab, "Thermocouples, Thermistors \& RTDs", Sensor Scientific, Inc., Fairfield, New Jersey.
} 
listed, YSI ${ }^{11}$ was happy to work with us and was the company we chose for the thermistor assemblies. YSI thermistors are also being considered for temperature measurements in the tunnel ${ }^{12}$.

YSI makes a number of different types of thermistors. We require high stability, so we chose the 46000 series of glass encapsulated, long term stability thermistors. The thermistors of this series can be purchased pre-sorted into either $0.2^{\circ} \mathrm{C}, 0.1^{\circ} \mathrm{C}$ or, $0.05^{\circ}$ $\mathrm{C}$ interchangeability families. Since none of these families meet our accuracy requirement out of the box, each sensor will need to be individually calibrated. We chose the less expensive $0.2^{\circ} \mathrm{C}$ interchangeability family. Note that the interchangeability specification has no bearing on the accuracy of a thermistor after it is individually calibrated. Within the 46000 series, the thermistor resistance is the next choice. It turns out that self heating errors are smallest for large resistance values, as will be discussed below, so we chose the 30 $\mathrm{K} \Omega$ thermistor with YSI part number 46008.

The probe that the thermistor is assembled in has part number YSI034. This probe has a non-magnetic 316 stainless steel tubular end which is 0.188 inches in diameter and 4 inches long. The leads have a hermetic seal allowing us to use the probe in a water bath for calibration without the danger of water entering the probe and degrading the performance. The leads are 6 feet long and we will place a connector on the end to attach to cables in the measurement system. A sample YSI034 probe assembly with 46008 thermistor is shown in figure 1.

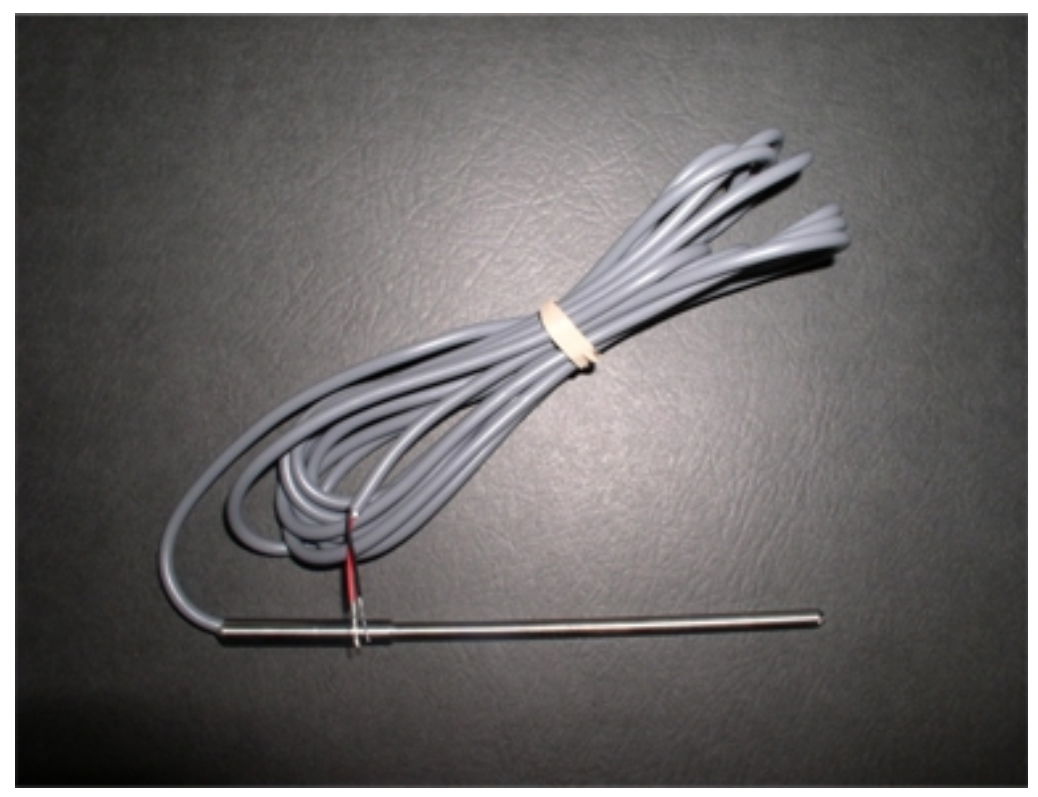

Figure 1: Sample YSI034 thermistor probe assembly.

The tubular thermistor assembly will be attached to an undulator with a mounting block. A schematic of the thermistor mount is shown in figure 2. The block is attached

\footnotetext{
${ }^{11}$ YSI Incorporated, Yellow Springs, Ohio, www.ysi.com.

${ }^{12}$ Wesley Craddock, SLAC, private communication.
} 
to the undulator with a bolt. The thermistor is inserted into a hole in the block. A layer of insulation covers the assembly. The block contains a second hole so that a reference thermometer can be inserted at any time to check the thermistor reading.
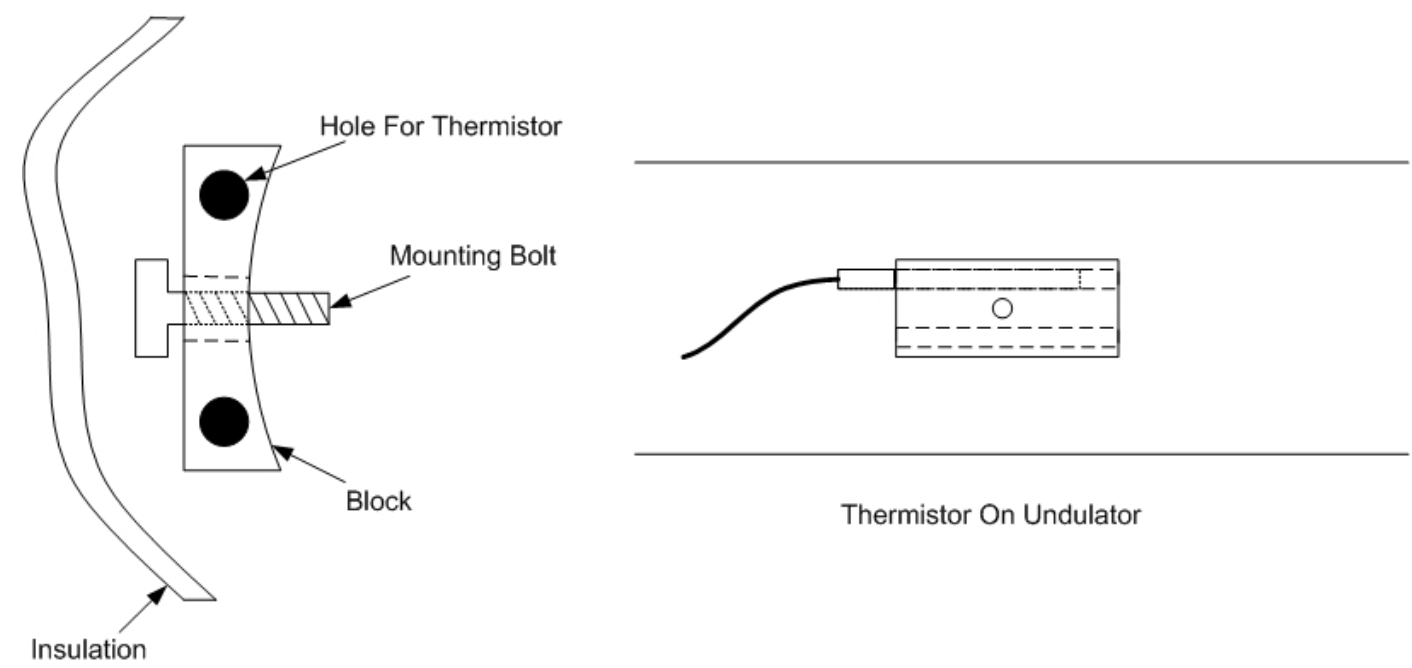

Figure 2: A mounting block attaches the thermistor probe to the undulator.

Once we have chosen thermistors as our sensors, the DMM and multiplexer selection can be made. The Agilent 34970A data acquisition/switch unit combines the DMM and multiplexer into a single instrument with adequate accuracy, as will be shown. It does offset compensation on 4 -wire resistance measurements and only uses $10 \mu \mathrm{A}$ on the $100 \mathrm{~K} \Omega$ range, keeping the thermistor self heating to an acceptable value as discussed below. The Agilent 34970A together with thermistor sensors provides a very cost effective system.

We will also have a pressure sensor and humidity sensor in the system. The pressure sensor will be a Vaisala PTB100 series analog barometer and the humidity sensor will be a Vaisala HMP45A probe. These sensors were used in the Sector 10 prototype system with success. We will purchase inexpensive calibration equipment for these sensors. Pressure and humidity are not expected to have a large effect on the magnetic measurements and are included in the system for completeness.

The temperature calibration system uses several pieces of equipment from Hart Scientific $^{13}$. A water bath, model 7320, keeps the thermistor under test and a reference thermometer, model 1504 with model 5642-D probe, at the same temperature. The reference thermometer is returned to the factory periodically for calibration. A triple point of water cell, model 5901, verifies the stability of the reference thermometer between calibrations. One or more very high stability reference resistors with small temperature coefficient, Precision Resistor Company ${ }^{14}$ model HR186N with resistance $30 \mathrm{~K} \Omega$, is used to monitor the stability of the DMM between calibrations.

\footnotetext{
${ }^{13}$ Hart Scientific, American Fork, Utah, www.hartscientific.com.

${ }^{14}$ Precision Resistor Co., Inc., Largo, Florida, www.precisionresistor.com.
} 


\subsection{System Configuration}

A schematic of the temperature measurement system is shown in figure 3. An electronics

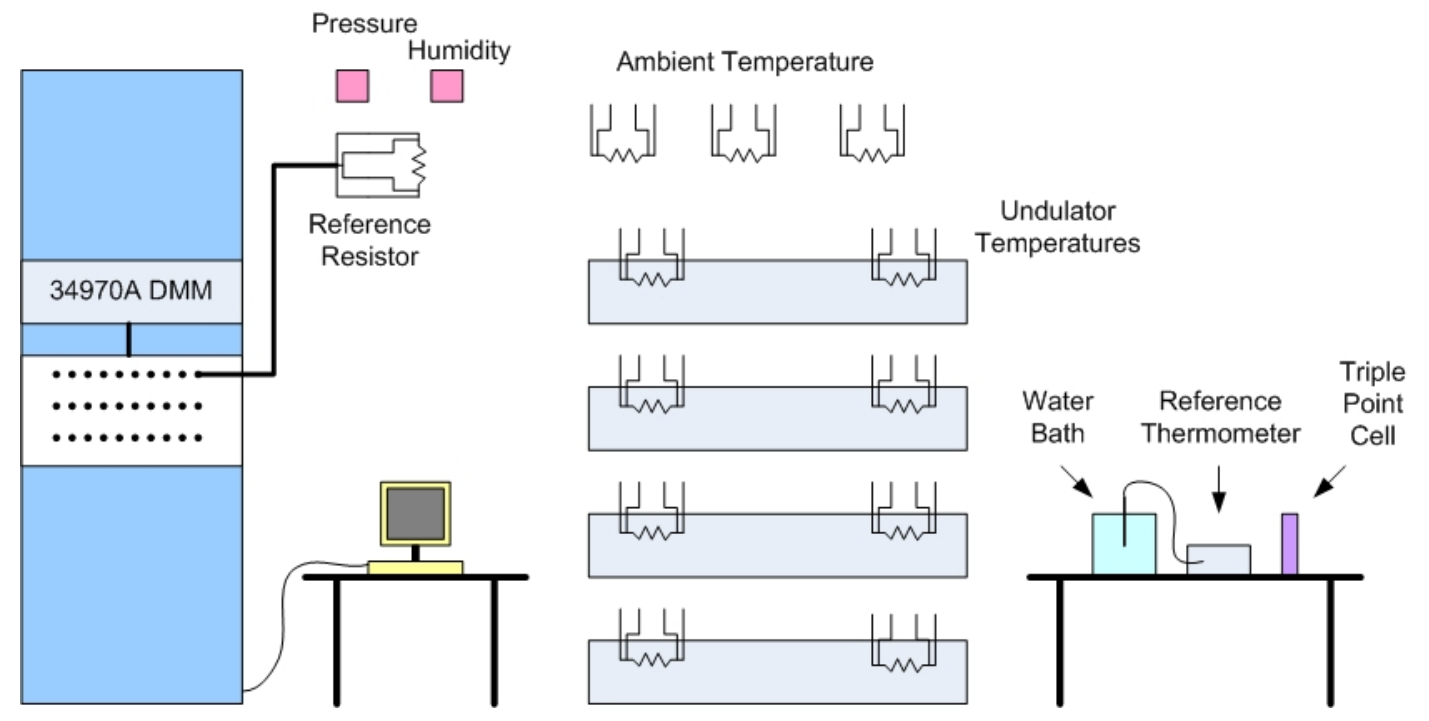

Figure 3: Schematic of the temperature measurement system.

rack contains the Agilent 34970A DMM/multiplexer and a connection panel where all sensors are attached. The connection panel and multiplexer are wired for 4 -wire resistance measurements. With this configuration, other resistance sensors such as RTDs can be included in the system, if desired. Voltages from the pressure and humidity sensors can still be measured with this wiring scheme.

A computer controls the Agilent 34970A to constantly measure sensor outputs. The computer applies calibrations in software to get the desired physical quantity. Data are stored over the network with all other measurements from each particular undulator.

The calibration system consists of a water bath and a reference thermometer with a NIST traceable calibration which acts as a secondary standard. Thermistor resistance as a function of temperature is measured to perform the calibration. A triple point of water cell is used to verify the stability of the reference thermometer between calibrations. A reference resistor is used to verify the stability of the Agilent 34970A.

\subsection{Software}

The measurement program for the system, written in LabWindows/CVI ${ }^{15}$, has been completed and tested. The user interface consists of tabbed sheets, one for the ambient measurements and one for each undulator. A setup area is included on each sheet to let the system know which sensor is being used for each measurement. Calibration data are stored in files, one for each sensor. The temperature history is displayed on each sheet. Temperature data for each undulator are stored with the magnetic measurement data for the

\footnotetext{
${ }^{15}$ National Instruments, Austin, Texas, www.ni.com.
} 
undulator.

\section{Error Analysis of the Temperature Measurement System}

There are a number of error sources in the temperature measurement system. In this section we discuss the errors and estimate their size.

\subsection{Thermistor Self Heating}

To measure the resistance of a thermistor, the DMM sends a current through the thermistor and measures the voltage across it. Power is delivered to the thermistor: $P=I^{2} R$ where $P$ is the power dissipated in the thermistor, $I$ is the excitation current, and $R$ is the thermistor resistance. The power heats the thermistor and causes a temperature measurement error. DMMs have different excitation currents for different resistance ranges. In order to estimate the optimal thermistor resistance, we calculate the power delivered to a resistor in the middle of each range of the Agilent 34970A in table 1. We see that with the excitation currents

\begin{tabular}{|l|l|l|l|}
\hline Range & Current & Resistance & Power \\
\hline $100 \Omega$ & $1 \mathrm{~mA}$ & $50 \Omega$ & $5 \times 10^{-5} \mathrm{~W}$ \\
\hline $1 \mathrm{~K} \Omega$ & $1 \mathrm{~mA}$ & $500 \Omega$ & $5 \times 10^{-4} \mathrm{~W}$ \\
\hline $10 \mathrm{~K} \Omega$ & $100 \mu \mathrm{A}$ & $5 \mathrm{~K} \Omega$ & $5 \times 10^{-5} \mathrm{~W}$ \\
\hline $100 \mathrm{~K} \Omega$ & $10 \mu \mathrm{A}$ & $50 \mathrm{~K} \Omega$ & $5 \times 10^{-6} \mathrm{~W}$ \\
\hline $1 \mathrm{M} \Omega$ & $5 \mu \mathrm{A}$ & $500 \mathrm{~K} \Omega$ & $1.25 \times 10^{-5} \mathrm{~W}$ \\
\hline
\end{tabular}

Table 1: Power delivered to various resistors by the Agilent 34970A.

given, the $100 \mathrm{~K} \Omega$ range delivers the least power to a thermistor in the middle of the range. Of the high stability grade thermistor resistance choices available from YSI, the $30 \mathrm{~K} \Omega$ thermistor will have the lowest self heating with the Agilent 34970A. This was a primary criterion for choosing the $30 \mathrm{~K} \Omega$ model number 46008 thermistor.

Thermistor companies provide a dissipation constant which is the power required to heat the thermistor by $1^{\circ} \mathrm{C}$. The dissipation constant for the 46000 series thermistors is $D=4 \mathrm{~mW} /{ }^{\circ} \mathrm{C}$ in air. The steady state temperature rise is $\Delta T=P / D$. For the model 46008 thermistor and the Agilent 34970A, $P=3 \times 10^{-6} \mathrm{~W}$ and the temperature rise is $\Delta T=\left(3 \times 10^{-6} \mathrm{~W}\right) /\left(4 \times 10^{-3} \mathrm{~W} /{ }^{\circ} \mathrm{C}\right)=7.5 \times 10^{-4}{ }^{\circ} \mathrm{C}$. This is well below our $0.01^{\circ} \mathrm{C}$ accuracy limit.

\subsection{DMM Accuracy}

The Agilent 34970A DMM has an accuracy specification for most ranges of $\Delta R=(0.01 \% \times$ reading $)+$ $(0.001 \% \times$ range $)$ over one year and over a temperature range of $23^{\circ} \mathrm{C} \pm 5^{\circ} \mathrm{C}$. A resistance measurement error is related to a temperature measurement error through the sensitivity $\alpha=\left(\frac{1}{R}\right)\left(\frac{d R}{d T}\right)$. The temperature error is given by $\Delta T=\Delta R / \alpha R$. The DMM resistance error causes a temperature error of

$$
\Delta T=\frac{1}{\alpha R}[(0.0001 R)+(0.00001 \times \text { range })]
$$


Rearranging terms, we get

$$
\Delta T=\frac{1}{\alpha}\left[(0.0001)+\left(0.00001 \times \frac{\text { range }}{R}\right)\right]
$$

Note that this expression is fairly insensitive to the sensor resistance used, so our choice of thermistor is not affected by the DMM resistance measurement error. For the YSI model 46008 thermistors at $20^{\circ} \mathrm{C}, \alpha \simeq-0.0453 \frac{1}{{ }^{\circ} \mathrm{C}}, R=30 \mathrm{~K} \Omega$, and the range is $100 \mathrm{~K} \Omega$. This gives a temperature measurement error of $\Delta T=2.94 \times 10^{-3}{ }^{\circ} \mathrm{C}$ from the DMM resistance measurement error. This is smaller than our $0.01{ }^{\circ} \mathrm{C}$ accuracy limit. The Agilent $34970 \mathrm{~A}$ is an adequate DMM for our measurements.

The DMM resistance measurement accuracy specification is for one year, necessitating yearly calibrations. To check for unforeseen DMM drift, we include one or more very stable reference resistors in the system. The Ultra Precision series resistors from Precision Resistor Co. have a drift of less than $0.001 \%$ per year $\left(\Delta T=2.2 \times 10^{-4}{ }^{\circ} \mathrm{C}\right.$ equivalent temperature error) and a temperature coefficient of $5 \mathrm{ppm}$ per degree Celsius. By monitoring the measurements of the stable reference resistor, we can monitor the drift of the DMM.

\subsection{Lead Length/Connectors}

When measuring sensor resistance, one must be concerned about the added resistance of the leads and connectors. Using 4-wire resistance measurements eliminates this problem.

One must also be concerned about thermal emfs in the circuit. The Agilent 34970A has an offset compensation feature to eliminate this problem. The voltage is measured with no current and then subtracted from the voltage measured in the resistance measurement.

\subsection{Thermistor Ageing}

Modern high stability thermistors are extremely stable. The YSI 46000 series thermistors have a specified drift of less than $0.01^{\circ} \mathrm{C}$ over 100 months at $25^{\circ} \mathrm{C}$. We expect very stable performance and we will check the thermistor calibrations periodically. We assign an error of $0.001^{\circ} \mathrm{C}$ for the thermistor drift between calibrations.

\subsection{Reference Thermometer Error}

To calibrate a thermistor, a reference thermometer is placed in a water bath with the thermistor and the thermistor resistance is recorded at a number of temperatures. The specified accuracy of the Hart Scientific model 1504 thermometer readout is $0.002^{\circ} \mathrm{C}$. The accuracy of the model $5642-\mathrm{D}$ probe is $0.001^{\circ} \mathrm{C}$. Together, these errors produce a reference thermometer error of $0.0022^{\circ} \mathrm{C}$.

The reference thermometer is a secondary standard with a NIST-traceable calibration. We will return it to the factory yearly for calibration. In order to check for drift in the reference thermometer between calibrations, we use a triple point of water cell. This cell provides a repeatable temperature to better than $0.0001^{\circ} \mathrm{C}$. The temperature of the triple point of water is defined to be $0.01^{\circ} \mathrm{C}$. Any significant difference we measure from this value using the reference thermometer will indicate a reference thermometer drift and the unit will be returned to the factory for calibration. 


\subsection{Water Bath Uniformity}

The Hart Scientific model 7320 water bath has a temperature uniformity specification of $\pm 0.005^{\circ} \mathrm{C}$. We will insert the thermistor probe and reference thermometer into a copper block in the water bath to further reduce the temperature difference between them. In addition, measurements can be made of the temperature at different points on the copper block to correct for temperature gradients. For our error analysis, we will include a worst case $\pm 0.005^{\circ} \mathrm{C}$ error to account for the thermistor and reference thermometer probe temperature difference during calibration.

\section{Calibration}

A thermistor is calibrated by placing it and the reference thermometer in a copper block in a water bath. The water bath allows temperature adjustment and keeps the thermistor and reference thermometer at approximately the same temperature. The copper block insures good thermal contact and reduces the small gradients and fluctuations of the water temperature in the bath. The resistance of the thermistor is recorded as a function of the reference thermometer temperature as the water bath temperature is changed and equilibrium is re-established.

It has been found experimentally that, with high accuracy, the thermistor resistance $R$ is related to temperature $T$ in degrees Kelvin by the Steinhart-Hart equation ${ }^{16}$

$$
\frac{1}{T}=A+B \ln (R)+C \ln (R)^{3}
$$

This parametrization simplifies the calibration process because only three calibration points are needed to determine the $A, B$, and $C$ parameters. The Steinhart-Hart equation is motivated as follows. Even though the thermistor is a ceramic, we model it as a semiconductor. The intrinsic carrier density is

$$
n_{i}=K e^{-E_{g} / 2 k T}
$$

where $K$ is a constant, $E_{g}$ is the band gap energy, and $k$ is Boltzmann's constant ${ }^{17}$. The resistance of the thermistor is proportional to $n_{i}$, so

$$
R=R_{0} e^{-E_{g} / 2 k T}
$$

This leads to the relation

$$
\ln (R)=A^{\prime}+B^{\prime} \frac{1}{T}
$$

where $A^{\prime}$ and $B^{\prime}$ are constants. This can be inverted to give

$$
\frac{1}{T}=A+B \ln (R)
$$

This simple model gives the basic relation between temperature and resistance. Steinhart and Hart improved the accuracy of this expression by adding correction terms in the form

\footnotetext{
${ }^{16}$ See, for instance, "Thermistor Application Notes", Betatherm Corp., Shrewsbury, MA, www.betatherm.com.

${ }^{17}$ B. Streetman and S. Banerjee, Solid State Electronic Devices, fifth edition, Prentice Hall, 2000.
} 
of a polynomial expansion, leading to equation 3. Second, fourth, and higher order terms can be added and their importance is discussed in a Thermometrics application note ${ }^{18}$. The application note suggests that equation 3 gives interpolation errors less than $0.003^{\circ} \mathrm{C}$ over $50^{\circ} \mathrm{C}$ spans. Over our small temperature range, equation 3 should be adequate, but we can test it by going to temperatures between our calibration points and comparing the parametrization to the reference thermometer temperature.

The calibration proceeds as follows. Three temperatures are chosen as calibration points, for example, $T_{1}=15^{\circ} \mathrm{C}=288.15^{\circ} \mathrm{K}, T_{2}=20^{\circ} \mathrm{C}=293.15^{\circ} \mathrm{K}, T_{3}=25^{\circ} \mathrm{C}$ $=298.15^{\circ} \mathrm{K}$. The resistance of the thermistor is measured at each temperature $R_{1}, R_{2}$, and $R_{3}$. This leads to the following set of equations expressed in matrix form

$$
\left[\begin{array}{c}
\frac{1}{T_{1}} \\
\frac{1}{T_{2}} \\
\frac{1}{T_{3}}
\end{array}\right]=\left[\begin{array}{lll}
1 & \ln \left(R_{1}\right) & \ln \left(R_{1}\right)^{3} \\
1 & \ln \left(R_{2}\right) & \ln \left(R_{2}\right)^{3} \\
1 & \ln \left(R_{3}\right) & \ln \left(R_{3}\right)^{3}
\end{array}\right]\left[\begin{array}{c}
A \\
B \\
C
\end{array}\right]
$$

The $A, B$, and $C$ parameters are found by inverting the matrix

$$
\left[\begin{array}{l}
A \\
B \\
C
\end{array}\right]=\left[\begin{array}{lll}
1 & \ln \left(R_{1}\right) & \ln \left(R_{1}\right)^{3} \\
1 & \ln \left(R_{2}\right) & \ln \left(R_{2}\right)^{3} \\
1 & \ln \left(R_{3}\right) & \ln \left(R_{3}\right)^{3}
\end{array}\right]^{-1}\left[\begin{array}{c}
\frac{1}{T_{1}} \\
\frac{1}{T_{2}} \\
\frac{1}{T_{3}}
\end{array}\right]
$$

Once $A, B$, and $C$ are known, a resistance measurement of the thermistor can be used to calculate the temperature using equation 3. Note that if more terms in the polynomial expansion are included, this same calibration procedure can still be used, but with more calibration measurements and larger matrices. Also note that other fitting methods can be used such as taking many calibration measurements and performing least squares fits to the $1 / T$ vs $\ln (R)$ data. Experience will dictate whether such measures are necessary.

During the calibration, both the reference thermometer temperature and the thermistor resistance are measured with errors. In addition, temperature gradients exist between the thermistor and the reference probe. This leads to errors in the $A, B$, and $C$ parameters. To estimate the resulting measurement error, we find the equivalent temperature error at a calibration point and assign this error to future measurements as the error component coming from calibration errors. The procedure is illustrated in figure 4. At a calibration point, the equivalent temperature error coming from the DMM resistance error is $0.00294^{\circ}$ $\mathrm{C}$, as discussed above. The reference thermometer temperature measurement error is $0.0022^{\circ} \mathrm{C}$. The thermistor self heating error is $0.00075^{\circ} \mathrm{C}$. The error from temperature gradients in the water bath is $0.005^{\circ} \mathrm{C}$. These four errors are added in quadrature to get an equivalent temperature error of $0.0062^{\circ} \mathrm{C}$ on each calibration point. This error shifts the parametrization up and down along the temperature axis in the worst case as shown in figure 4. Shifts in the parametrization cause shifts in the temperature measurements.

Figure 4 also illustrates how the Steinhart-Hart parametrization is different than the actual relation between temperature and resistance. This discrepancy is the interpolation error mentioned above and an error estimate of $0.003^{\circ} \mathrm{C}$ was assigned to it.

\footnotetext{
18 "NTC Thermistors", Thermometrics, Inc. Edison, New Jersey, www.thermometrics.com.
} 


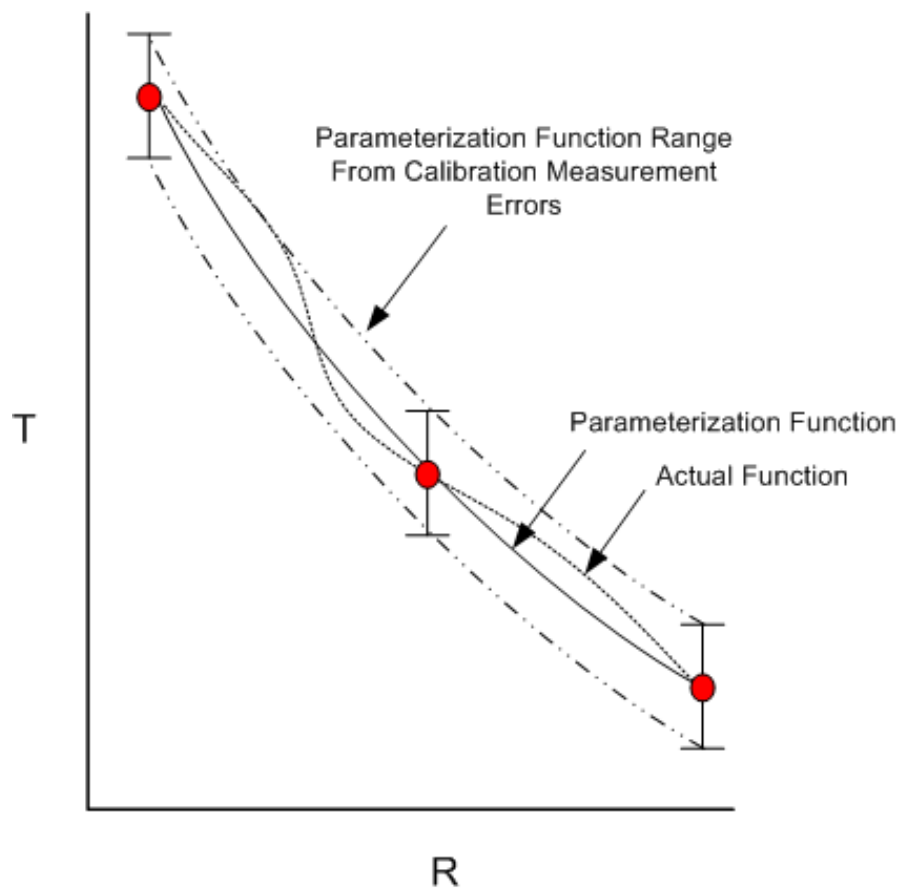

Figure 4: Effects of errors in the calibration measurements and interpolation errors.

\section{Temperature Measurement Error Summary}

A summary of the errors for a thermistor temperature measurement is given in table 2 .

\begin{tabular}{|l|l|}
\hline Error & Value \\
\hline Thermistor calibration, errors on calibration points & $0.0062^{\circ} \mathrm{C}$ \\
\hline Thermistor calibration, interpolation error & $0.003^{\circ} \mathrm{C}$ \\
\hline Thermistor self heating & $0.00075^{\circ} \mathrm{C}$ \\
\hline DMM resistance measurement error & $0.00294^{\circ} \mathrm{C}$ \\
\hline Lead/connector added resistance & $0.00^{\circ} \mathrm{C}$ \\
\hline Thermal EMFs & $0.00^{\circ} \mathrm{C}$ \\
\hline Thermistor ageing & $0.001^{\circ} \mathrm{C}$ \\
\hline
\end{tabular}

Table 2: Summary of the temperature measurement errors.

Adding these errors in quadrature gives an overall error for each measurement of $\Delta T=$ $0.008^{\circ} \mathrm{C}$. This is our estimate of the measurement system accuracy. This will be checked by placing the reference thermometer in good thermal contact with each thermistor probe during operation and comparing answers. 


\section{Conclusion}

A temperature measurement system is being developed for the MMF. The system is based on a prototype system in the Sector 10 alignment lab at SLAC which has operated successfully for over two years. Temperature measurement accuracy in the MMF must be better than $\pm 0.01^{\circ} \mathrm{C}$ and the system under development is expected to meet this accuracy requirement.

Acknowledgements

We wish to thank Jim Welch and Wes Craddock for valuable discussions. 